\title{
Transport in Two-Dimensional Disordered Semimetals
}

\section{Citation}

Knap, Michael, Jay D. Sau, Bertrand I. Halperin, and Eugene Demler. 2014. “Transport in TwoDimensional Disordered Semimetals." Physical Review Letters 113 (18) (October). doi:10.1103/ physrevlett.113.186801.

\section{Published Version}

10.1103/PhysRevLett.113.186801

\section{Permanent link}

http://nrs.harvard.edu/urn-3:HUL.InstRepos:22856735

\section{Terms of Use}

This article was downloaded from Harvard University's DASH repository, and is made available under the terms and conditions applicable to Other Posted Material, as set forth at http:// nrs.harvard.edu/urn-3:HUL.InstRepos:dash.current.terms-of-use\#LAA

\section{Share Your Story}

The Harvard community has made this article openly available.

Please share how this access benefits you. Submit a story.

Accessibility 


\title{
Transport in Two-Dimensional Disordered Semimetals
}

\author{
Michael Knap, ${ }^{1,2, *}$ Jay D. Sau, ${ }^{1,3}$ Bertrand I. Halperin, ${ }^{1}$ and Eugene Demler ${ }^{1}$ \\ ${ }^{1}$ Department of Physics, Harvard University, Cambridge, Massachusetts 02138, USA \\ ${ }^{2}$ ITAMP, Harvard-Smithsonian Center for Astrophysics, Cambridge, Massachusetts 02138, USA \\ ${ }^{3}$ Department of Physics, Condensed Matter Theory Center and the Joint Quantum Institute, \\ University of Maryland, College Park, Maryland 20742, USA
}

(Received 21 May 2014; published 27 October 2014)

\begin{abstract}
We theoretically study transport in two-dimensional semimetals. Typically, electron and hole puddles emerge in the transport layer of these systems due to smooth fluctuations in the potential. We calculate the electric response of the electron-hole liquid subject to zero and finite perpendicular magnetic fields using an effective medium approximation and a complementary mapping on resistor networks. In the presence of smooth disorder and in the limit of a weak electron-hole recombination rate, we find for small but finite overlap of the electron and hole bands an abrupt upturn in resistivity when lowering the temperature but no divergence at zero temperature. We discuss how this behavior is relevant for several experimental realizations and introduce a simple physical explanation for this effect.
\end{abstract}

In semimetals both electrons and holes contribute to transport. Typical examples are indirect bulk semiconductors with a small band overlap. More recently also two-dimensional systems, including $\mathrm{HgTe}$ quantum wells close to the topological insulator to metal transition [1-5], BiSe thin films [6], and bilayer graphene [7,8], have been identified to exhibit semimetallic properties. Electron and hole puddles typically emerge in the transport layer of these systems due to disorder that varies smoothly in space on a scale that is large compared to the mean free path of the charge carriers.

In this Letter, we introduce a two-fluid model to explore the effects of smooth disorder on the transport properties of two-dimensional electron-hole mixtures, Fig. 1. The model assumes that as a result of long-range correlated disorder, and a small intrinsic overlap between the electron and hole band, the sample at low temperatures can be divided into three types of regions: areas where only electron states or only hole states are occupied, and intermediate areas where both types of carriers are occupied, Fig. 1(c). We assume that carriers are scattered easily within a band, due to phonons or residual impurities, but that recombination between electrons and holes is suppressed. Then, if neither the pure electron regions nor the pure hole regions percolate across the sample, charge transport at low temperatures may be effectively limited by the relatively narrow percolating portion where both electrons and holes are occupied and each carrier type has a low density. As we shall see, this can lead to an anomalously high resistivity at low temperatures.

To make our picture quantitative, we introduce a model with smooth disorder, and obtain the local densities of electrons and holes using a Thomas-Fermi-like approximation. We assume that the electron and hole mobilities differ from each other, but are independent of the respective carrier densities. We solve the resulting inhomogeneous, two-component conductance problem using an effective medium approximation (EMA) [9-14]. The EMA has already been used successfully to characterize transport in GaAs quantum wells, where smooth disorder has been identified as the main mechanism for the transition from metallic to insulating behavior as a function of electron

(a)

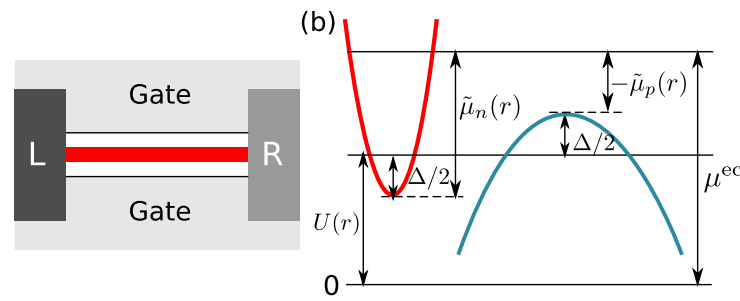

(c)

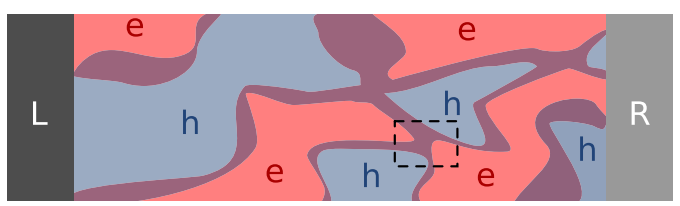

FIG. 1 (color online). (a) A two-dimensional electron and hole mixture, red region, is driven by a bias voltage imprinted from the potential difference in the left and the right lead (side view). (b) Definitions of the energy scales: $\Delta$ is the distance between the conduction and the valance band edge, which is taken to be negative when they overlap. $\mu^{\mathrm{ec}}$ is the electrochemical potential, which at equilibrium is identical for electrons and holes. The electrical potential $U(r)$ and thus also the chemical potentials of the electrons $\tilde{\mu}_{n}(r)$ and holes $\tilde{\mu}_{p}(r)$ vary smoothly in space due to the disorder. (c) The long-range fluctuations in the potential create regions where only electrons (red) or only holes (blue) are occupied, and regions where both carriers coexist (purple) (top view). 
density [15-22]. We investigate dependences of the transport on band overlap, temperature, gate voltage, electron-hole recombination rate, and magnetic field, using parameters we believe appropriate to the $\mathrm{HgTe}$ quantum wells in Ref. [5]. In the case of zero magnetic field, we checked the validity of the EMA by introducing a model of resistors on a discrete lattice, which we solve numerically.

Quantum well at equilibrium.-We consider a twodimensional electron and hole mixture with densities $n_{n}(r)$ and $n_{p}(r)$, respectively. The characteristic correlation length scale of the disorder is set by the distance to charged impurities, which can typically be on the order of hundred nanometers. We expect this scale to be much larger than both the microscopic mean-free path of the charge carriers and the Coulomb screening length. Therefore, we will take a model in which we treat Coulomb interactions to be of the local form

$$
\begin{gathered}
\phi_{n}(r)=\int d r^{\prime} K_{c}\left(r-r^{\prime}\right) n_{n}\left(r^{\prime}\right)=K n_{n}(r), \\
\phi_{p}(r)=-\int d r^{\prime} K_{c}\left(r-r^{\prime}\right) n_{p}\left(r^{\prime}\right)=-K n_{p}(r),
\end{gathered}
$$

where $K_{c}\left(r-r^{\prime}\right)$ is the Coulomb kernel and $K$ is the effective Coulomb interaction parameter. The precise length scales of the screened Coulomb interaction and disorder potential do not enter into our analysis.

We define the energies in our description according to the level scheme illustrated in Fig. 1(b):

$$
\begin{gathered}
\mu_{n}^{\mathrm{ec}}=\tilde{\mu}_{n}(r)+\frac{\Delta}{2}+U(r), \\
\mu_{p}^{\mathrm{ec}}=-\tilde{\mu}_{p}(r)-\frac{\Delta}{2}+U(r),
\end{gathered}
$$

where $\mu_{n}^{\mathrm{ec}}\left(\mu_{p}^{\mathrm{ec}}\right)$ is the electrochemical potential of the electrons (holes), $\tilde{\mu}_{n}(r)\left[-\tilde{\mu}_{p}(r)\right]$ is the electron [hole] chemical potential measured from the bottom of the conduction band [top of the valance band], and $\Delta$ is the distance between the edges of the respective bands. Finite band overlap as indicated in Fig. 1(b) corresponds to $\Delta<0$. The electrical potential is defined as $U(r)=\phi_{n}(r)+$ $\phi_{p}(r)+V(r)$, where $V(r)$ describes the smooth spatial randomness of the potential on scale $W$, which gives rise to the puddle formation, Fig. 1(c).

At equilibrium the electrochemical potentials of the electrons and holes are identical, i.e., $\mu_{n}^{\mathrm{ec}}=\mu_{h}^{\mathrm{ec}}=\mu^{\mathrm{ec}}$ and determined by the gate voltage. Using Eqs. (1) and (2) we find

$$
\begin{gathered}
\tilde{\mu}_{n}(r)=\mu^{\mathrm{ec}}-K\left[n_{n}(r)-n_{p}(r)\right]-V(r)-\frac{\Delta}{2}, \\
\tilde{\mu}_{p}(r)=-\mu^{\mathrm{ec}}+K\left[n_{n}(r)-n_{p}(r)\right]+V(r)-\frac{\Delta}{2},
\end{gathered}
$$

which have to be solved self-consistently in the presence of disorder as $n_{\alpha}(r)$ itself depends on the chemical potential $\tilde{\mu}_{\alpha}(r)$.

Nonequilibrium treatment.-When a bias voltage is applied to the electrodes at the edges of the sample, the local potential $\delta U(r)$ changes in the entire sample, thus causing a change of $\delta \phi_{\alpha}(r)$ and $\delta \mu_{\alpha}^{\mathrm{ec}}(r)$. Out of equilibrium the bulk electron and hole electrochemical potentials therefore differ from each other:

$$
\begin{gathered}
\delta \mu_{n}^{\mathrm{ec}}(r)=\delta \tilde{\mu}_{n}(r)+\delta U(r), \\
\delta \mu_{p}^{\mathrm{ec}}(r)=-\delta \tilde{\mu}_{p}(r)+\delta U(r) .
\end{gathered}
$$

At the boundary the electrochemical potentials of both components are identical and fixed by the potential imprinted from leads $\delta \mu^{\text {ext }}$.

The electron and hole currents are driven by the electrochemical potentials

$$
\left(\begin{array}{c}
j_{n} \\
j_{p}
\end{array}\right)=-\Sigma\left(\begin{array}{l}
\nabla \delta \mu_{n}^{\mathrm{ec}} \\
\nabla \delta \mu_{p}^{\mathrm{ec}}
\end{array}\right), \quad \Sigma=\left(\begin{array}{cc}
\sigma_{n} & 0 \\
0 & \sigma_{p}
\end{array}\right),
$$

where $\sigma_{\alpha}(r)$ is the microscopic conductivity whose functional form we derive in the Supplemental Material from a Boltzmann transport formalism [23]. In particular, we consider the linear current response of the two fluids to a small bias voltage. In that regime energy relaxation effects have not to be considered and thus we can assume that the system is locally at equilibrium. In our notation, $j_{n}$ and $j_{p}$ are vectors in the $x-y$ plane, while $\sigma_{\alpha}$ are scalars, in the absence of an applied magnetic field.

The nonequilibrium dynamics of the electron-hole channels is decoupled, Eq. (5). However, recombination processes of rate $\gamma$ dynamically couple the fluids, which can be taken into account by the continuity equation

$$
\frac{\partial \vec{n}}{\partial t}+\nabla \vec{j}=\Gamma \delta \vec{\mu}^{\mathrm{ec}}, \quad \Gamma=\left(\begin{array}{cc}
-\gamma & \gamma \\
\gamma & -\gamma
\end{array}\right) .
$$

Here, we used a two-component vector notation with the electron and hole component at the first and second entry, respectively. The steady state is obtained from the continuity equation by setting $\partial \vec{n} / \partial t=0$ and boundary conditions that fix the electrochemical potential:

$$
\nabla \vec{j}-\Gamma \delta \vec{\mu}^{\mathrm{ec}}=0,\left.\quad \delta \mu_{n}^{\mathrm{ec}}\right|_{\mathrm{bnd}}=\left.\delta \mu_{p}^{\mathrm{ec}}\right|_{\mathrm{bnd}}=\delta \mu^{\mathrm{ext}} .
$$

Solving Eq. (7) amounts to determining the conductance of a random medium. One approach is to discretize Eq. (7) and map it onto a resistor network, see the Supplemental Material [23]. Alternatively, one can exploit approximate features of such problems by a mean-field treatment, often referred to as the EMA. 
Cheianov et al. [24] have employed a resistor network model, similar in some respects to the one introduced here, to derive the critical exponents and scaling behavior of graphene near its neutrality point. They use a percolation network analysis, which if applied to the present model, could be used to obtain the singular behavior in the limit where $T, \Delta$, and $\gamma$ tend to zero. In contrast, EMA predictions for the critical behavior would be qualitatively, but not quantitatively correct.

Effective medium approximation.-The EMA considers inclusions, labeled with the superscript $i$, that are embedded in an effective medium, labeled with the superscript $m$. The embedding is determined self-consistently by requiring that the current in the effective medium $\vec{j}^{m}=-\Sigma^{m}\left\langle\nabla \delta \vec{\mu}^{\mathrm{ec}}\right\rangle$ be identical to the average current in the sample $\left\langle\vec{j}^{i}\right\rangle=-\left\langle\Sigma^{i} \nabla \delta \vec{\mu}^{\mathrm{ec}}\right\rangle$, for details see the Supplemental Material [23].

The resistivity can be evaluated from the total current response of the system. Considering that the electrochemical potential at the boundary is fixed (7), we define the total resistivity $\rho$ as $j_{n}+j_{p}=\rho^{-1} \nabla \delta \mu^{\text {ext }}$ with

$$
\rho^{-1}=\sum_{\alpha \beta} \Sigma_{\alpha \beta}^{m},
$$

where $\Sigma^{m}$ is the self-consistently determined conductivity matrix of the medium.

Resistivity of disordered HgTe quantum wells.-We now apply the method developed for a general disordered twofluid model to the HgTe quantum wells studied experimentally in Refs. [1-5]. In HgTe quantum wells a transition from a topological trivial insulator to a quantum spin Hall insulator can be driven by enhancing the thickness of the well [25-27]. When further increasing the width of the well the system undergoes another transition to a semimetallic phase in which both electron and hole carriers contribute to transport [1-5]. In the following, we consider $20 \mathrm{~nm}$ HgTe quantum wells grown in the (100) direction as studied in Ref. [5]. In that system, the effective electron and hole masses are very different $m_{p} / m_{n} \sim 6$ [4], a unit cell contains one electron and four hole pockets, and at atmospheric pressure the conduction and valance band overlap by about $|\Delta| \sim 1.2$ to $1.5 \mathrm{meV}$. In the experiment of Ref. [5] hydrostatic pressure of $\sim 15 \mathrm{kbar}$ is applied to the sample, which is expected to decrease the band overlap.

In accordance with these observations we choose the following parameters for our model: we set the Coulomb interaction parameter to $K=0.5 \mathrm{~m}_{n}^{-1}$, take into account the large difference in the effective electron and hole masses $m_{p} / m_{n}=6$, and set $m_{n}=0.025$. We sample the local potential $V(r)$ from a uniform distribution of width $W=1$, which we use as the unit of energy. The disorder strength $W$ is renormalized by the effective screening parameter $\tilde{K}=1+g K$, where $g=\left(m_{n}+4 m_{p}\right) / \pi$, yielding the effective disorder strength $\tilde{W}=W / \tilde{K} \sim 0.2$. Long-range disorder will influence the transport provided the band overlap $|\Delta|<\tilde{W}$. Based on these considerations, we choose two extreme limits for the band overlap, $\Delta=-0.5$ and $\Delta=-0.025$, which should model zero and high pressure in experiment [5]. The studied HgTe quantum well has an indirect band structure in which the extrema of the conduction and valance bands are at different wave vectors, see Fig. 1(b). The electron-hole recombination would therefore require phonon scattering, which is suppressed at low temperatures. Thus, we mostly consider a zero carrier recombination rate $\gamma=0$.

In Fig. 2 we show the resistivity obtained from the EMA as a function of the gate voltage $V_{g}$, which directly modifies the electrochemical potential in Eq. (3). In accordance with the experiment [5] we observe metallic behavior for $\Delta=-0.5$, with a weak dependence on temperature only [Fig. 2(a)]. The asymmetry in the curves with respect to $V_{g}=0$ arises due to the large difference in electron and hole masses. For comparison we show the pure case $W=0$, dashed lines. For the reduced band overlap $\Delta=$ -0.025 and at $V_{g} \sim 0$, the resistivity increases strongly at low temperatures while at high temperatures the system remains conducting [Fig. 2(b)]. Further the maximum in the resistivity is shifted toward lower gate voltage. These results qualitatively explain several features of the $\mathrm{HgTe}$ quantum well experiments of Ref. [5].

The enhancement of the resistivity $\rho$ at low temperatures $T$ and the flattening out at high temperatures is demonstrated in Fig. 3 for a fixed gate voltage $V_{g}$. In this plot we compare the results obtained within the EMA to the solution of a resistor network [23] of size $L \times L=400 \times$ 400 for a vanishing electron-hole recombination rate $\gamma=0$ and find good agreement. Finite $\gamma>0$ decreases the sharp low-temperature feature. We also compare the resistivity of the disordered systems to the resistivity of the clean system and find an enhancement at low temperatures, which is one of the main observations of our work.
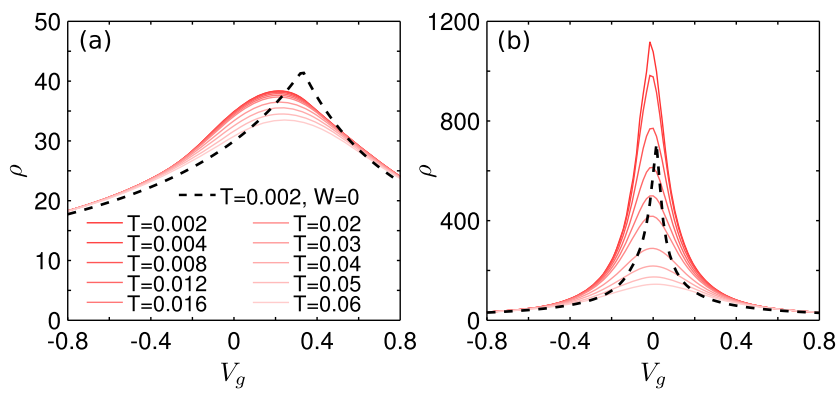

FIG. 2 (color online). Resistivity $\rho$, Eq. (8), as a function of the gate voltage $V_{g}$ for different temperatures $T$, characteristic scale of the potential fluctuations $W=1$, vanishing electron-hole recombination rate $\gamma=0$, and (a) band overlap $\Delta=-0.5$ and (b) $\Delta=-0.025$. Solid curves are evaluated for disordered systems using the effective medium approximation, while the dashed line is evaluated for a clean system. For a small band overlap (b) the random local potential leads to an increase of resistivity. 


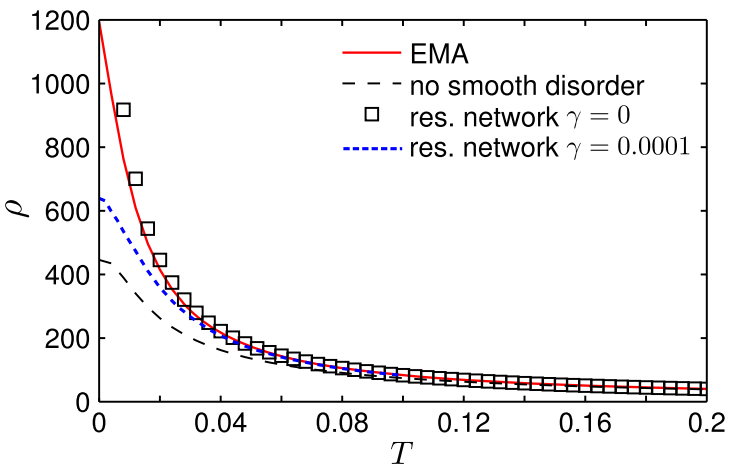

FIG. 3 (color online). Resistivity $\rho$ as a function of temperature $T$ for $V_{g}=-0.02, \Delta=-0.025, W=1$, and $\gamma=0$ evaluated with the EMA, solid line, and resistor networks, squares. These data are compared with the resistivity $\rho$ in the presence of a weak electron hole recombination rate $\gamma=0.0001$, dotted line, and of the clean system $W=0$, dashed line. At low temperatures $\rho$ is considerably enhanced by disorder.

A percolation picture provides further insights. When the recombination rate is very small, we must compute separately the conserved currents of electrons and holes, and add the results in the end. Let us consider the electron conductance as an example. If the band overlap is small, and the system is electrically neutral, then the regions where only electrons exist at $T=0$ will not percolate across the sample. In order to get from one of these regions to another, an electron has to cross the intermediate region, where electrons and holes coexist, which will generally occur at isolated junctions, where the two electron puddles come close together, see, e.g., the dashed rectangle in Fig. 1(c). The conductance of these junctions will be small, since they occur at places where the electron and hole densities are both nearly vanishing. The resistance of the electron network will be dominated by these junctions, and in fact it will diverge in the limit where the overlap goes to zero and there are equal numbers of electrons and holes. By contrast, at high temperatures, there will be a large number of thermally excited electrons and holes even in the regions separating the electron and hole dominated areas, so carriers can get across the sample without crossing a region of low conductivity. This physics is correctly captured by the EMA.

Resistivity in the presence of magnetic field.-To understand the relative electron and hole dominance we study the magnetotransport for fields applied perpendicular to the transport layer. The model we derived for a setting with zero magnetic field is readily generalized to finite magnetic fields, see the Supplemental Material [23], with the key difference that the resistivity has a tensorial structure consisting of a longitudinal $\rho_{x x}$ and a transverse $\rho_{x y}$ contribution $[28,29]$.

In Fig. 4 we show the (a) longitudinal $\rho_{x x}$ and the (b) transverse $\rho_{x y}$ resistivity as a function of magnetic field $B$ for fixed gate voltage $V_{g}=-0.16$. We find that the
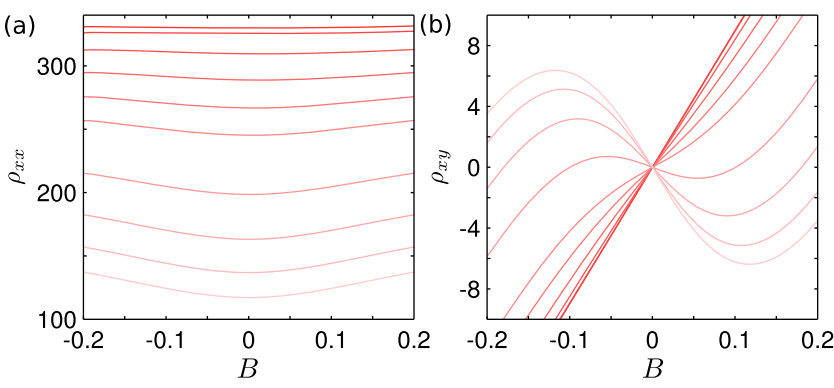

FIG. 4 (color online). (a) Longitudinal $\rho_{x x}$ and (b) transverse $\rho_{x y}$ resistivity as a function of the magnetic field $B$ applied perpendicular to an electron-hole mixture for the same temperatures $T$ as in Fig. 2. The curves are taken at gate voltage $V_{g}=-0.16$, band overlap $\Delta=-0.025$, disorder strength $W=1$, and relaxation rate $\gamma=0$.

longitudinal resistivity $\rho_{x x}$ increases with magnetic field $B$ and decreases with temperature $T$. For the chosen gate voltage the sign of the Hall charge (i.e., the slope of $\rho_{x y}$ at $B=0$ ) changes with temperature. The gate voltage is adjusted such that at low temperatures holes are the dominating charge carriers. With increasing temperature percolating paths open up faster for the light electrons as compared to the heavy holes. Thus, the electrons dominate transport at high temperatures leading to the change of the Hall charge.

Conclusions and outlook.-We developed a theory for transport in long-range disordered two-fluid systems as realized for instance in semimetallic quantum wells, thin films, and bilayer graphene.

We applied the developed technique to study transport in HgTe quantum wells and found that it captures several characteristic features observed in experiment [5] including the strong enhancement of the resistivity at low temperatures near charge neutrality. In Ref. [5] the authors proposed an alternative explanation of this effect based on the phase transition to an excitonic insulator at low temperatures, which does not consider long-range disorder but rather requires strong interactions. In contrast, in our theory, which identifies long-range disorder as a crucial mechanism, the sharp enhancement of the resistivity is not indicative of a true phase transition. At low temperatures the resistivity will rather saturate, albeit at a very large value. This feature is generic for semimetals with a small band overlap and results from (i) the relatively small percolating portion of coexisting electron and hole states and (ii) the vanishing electron-hole recombination rate relevant to the indirect band structures, such as the one of the considered HgTe quantum well.

This leads us to the conclusion that large length-scale disorder is a central mechanism in these experiments. Of course a complex interplay between long-range disorder and interactions is conceivable as well. Further experimental studies are therefore needed to fully confirm the picture. In particular, it would be interesting to explore the 
resistivity as a function of applied pressure, which should tune the band overlap continuously, and thus allow us to study the emergence of the strong enhancement of the resistivity at low temperatures.

We thank D. Kvon for introducing us to this problem and for sharing experimental results prior to publication. The authors acknowledge support from Harvard-MIT CUA, ARO-MURI Quism program, ARO-MURI on Atomtronics, STC Center for Integrated Quantum Materials, NSF Grant No. DMR-1231319, as well as the Austrian Science Fund (FWF) Project No. J 3361-N20.

*knap@ physics.harvard.edu

[1] Z. D. Kvon, E. B. Olshanetsky, D. A. Kozlov, N. N. Mikhailov, and S. A. Dvoretskii, JETP Lett. 87, 502 (2008).

[2] E. B. Olshanetsky, Z. D. Kvon, M. V. Entin, L. I. Magarill, N. N. Mikhailov, I. O. Parm, and S. A. Dvoretsky, JETP Lett. 89, 290 (2009).

[3] Z. D. Kvon, E. B. Olshanetsky, E. G. Novik, D. A. Kozlov, N. N. Mikhailov, I. O. Parm, and S. A. Dvoretsky, Phys. Rev. B 83, 193304 (2011).

[4] E. B. Olshanetsky, Z. D. Kvon, N. N. Mikhailov, E. G. Novik, I. O. Parm, and S. A. Dvoretsky, Solid State Commun. 152, 265 (2012).

[5] E. B. Olshanetsky, Z. D. Kvon, Y. A. Gerasimenko, V. Prudkoglyad, V. Pudalov, N. N. Mikhailov, and S. A. Dvoretsky, Pis'ma v ZhETF 98, 947 (2013).

[6] C. Kastl, T. Guan, X. Y. He, K. H. Wu, Y. Q. Li, and A. W. Holleitner, Appl. Phys. Lett. 101, 251110 (2012).

[7] B. E. Feldman, J. Martin, and A. Yacoby, Nat. Phys. 5, 889 (2009).

[8] E. McCann and M. Koshino, Rep. Prog. Phys. 76, 056503 (2013).

[9] D. A. G. Bruggeman, Ann. Phys. (Berlin) 416, 636 (1935).

[10] R. Landauer, J. Appl. Phys. 23, 779 (1952).
[11] D. Stroud, Phys. Rev. B 12, 3368 (1975).

[12] S. Kirkpatrick, Rev. Mod. Phys. 45, 574 (1973).

[13] E. Rossi, S. Adam, and S. Das Sarma, Phys. Rev. B 79, 245423 (2009).

[14] R. P. Tiwari and D. Stroud, Phys. Rev. B 79, 165408 (2009).

[15] S. V. Kravchenko, G. V. Kravchenko, J. E. Furneaux, V. M. Pudalov, and M. D'Iorio, Phys. Rev. B 50, 8039 (1994).

[16] Y. Meir, Phys. Rev. Lett. 83, 3506 (1999).

[17] S. Ilani, A. Yacoby, D. Mahalu, and H. Shtrikman, Phys. Rev. Lett. 84, 3133 (2000).

[18] Y. Meir, Phys. Rev. B 61, 16470 (2000).

[19] Y. Meir, Phys. Rev. B 63, 073108 (2001).

[20] S. Das Sarma, M. P. Lilly, E. H. Hwang, L. N. Pfeiffer, K. W. West, and J. L. Reno, Phys. Rev. Lett. 94, 136401 (2005).

[21] L. A. Tracy, E. H. Hwang, K. Eng, G. A. Ten Eyck, E. P. Nordberg, K. Childs, M. S. Carroll, M. P. Lilly, and S. Das Sarma, Phys. Rev. B 79, 235307 (2009).

[22] S. Das Sarma, E. H. Hwang, and Q. Li, Phys. Rev. B 88, 155310 (2013).

[23] See Supplemental Material at http://link.aps.org/ supplemental/10.1103/PhysRevLett.113.186801 for technical details on the transport coefficients, the EMA, and resistor networks.

[24] V. V. Cheianov, V. I. Fal'ko, B. L. Altshuler, and I. L. Aleiner, Phys. Rev. Lett. 99, 176801 (2007).

[25] B. A. Bernevig, T. L. Hughes, and S.-C. Zhang, Science 314, 1757 (2006).

[26] M. König, S. Wiedmann, C. Brüne, A. Roth, H. Buhmann, L. W. Molenkamp, X.-L. Qi, and S.-C. Zhang, Science 318, 766 (2007).

[27] S. Hart, H. Ren, T. Wagner, P. Leubner, M. Mühlbauer, C. Brüne, H. Buhmann, L. W. Molenkamp, and A. Yacoby, Nat. Phys. 10, 638 (2014).

[28] A. A. Abrikosov, Introduction to the Theory of Normal Metals (Academic Press, New York, 1972).

[29] D. J. Bergman and Y. M. Strelniker, Phys. Rev. B 60, 13016 (1999). 